\title{
Different shapes of tracks in phlogopite, biotite and soda lime glass
}

\author{
Mohan Singh, Lakhwant Singh* and Bikram Singh \\ Department of Physics, Guru Nanak Dev University, \\ Amritsar-143 005, India \\ E-mail : lakhwant@yahoo.com
}

\begin{abstract}
Etched track opening geometries in Biotite, Phlogopite and soda-lime glass irradiated with swift heavy ions $\left[{ }^{197} \mathrm{Au}(11.64 \mathrm{MeV} / \mathrm{n}),{ }^{136} \mathrm{Xe}(11.56 \mathrm{MeV} / \mathrm{n}),{ }^{58} \mathrm{Ni}(11.56 \mathrm{MeV} / \mathrm{n})\right]$ at different angle of incidence have been studied using appropriate chemical etching technique. Different geometries (Hexagonal, irregular polygon, triangular in case of Biotite and Phlogopite, and circular \& elliptical in case of soda lime glass detector) of heavy ion tracks are reported in the present investigations using optical microscope. The different shapes of these heavy ions track geometries are found to be related with various target-projectile parameters (viz : type of projectiles, energy, stopping power, angle of incidence of the projectile, density of defects and its reactivity with etchant, etching conditions and chemical structure of the detectors). The dependence of different shapes of heavy ion tracks in isotropic and anisotropic medium on the variation of radiation damage densities along the ion trajectories have also been discussed in the present paper.
\end{abstract}

Keywords : Heavy ions; track geometries; phlogopite; biotite; soda-lime glass

PACS Nos. : $61.72 . F f ; 61.80 . J h ; 91.60 . E d$

\section{Introduction}

Numerous studies on ion-beam science have focused on the morphology and mechanism of radiation damage in various classes of materials [1-5]. Probing the track structure is not only of academic interest where the nature of tracks is of fundamental importance but ion-track engineering may be developed to a stage allowing controlled fabrication of trackstructured nano-components for electrical and mechanical devices [6]. Chemically etched tracks in solid state nuclear track detectors have great applications in various field of science and technology e.g. radioprotection, medicine, biology, nano-technology [7-9]. In amorphous solid state detectors such as plastics and glasses, the only parameter that

\footnotetext{
* Corresponding Author
} 
affects the track opening shape is the angle of the projectile with the detector surface i.e. the dip angle $[10,11]$. By the variation of dip angle the eccentricity of the elliptical etch pit opening change. Because of the complexities involved in the structure of crystalline materials the track opening shapes in these detectors have complicated geometries. The range of the penetrating ions in these types of detectors (e.g. micas) depends on the angle of incidence of the ion because of the anisotropic effect of layered crystals [12]. Such studies can provide valuable information about the parameters affecting the track shapes that may be used in making microfilters using solid state nuclear track [13-16].

The track opening geometries of heavy ions in various detectors mainly depends on the nature of the detectors. In the present work, track opening geometries of heavy ions $\left[{ }^{197} \mathrm{Au}(11.64 \mathrm{MeV} / \mathrm{n}),{ }^{136} \mathrm{Xe}(11.56 \mathrm{MeV} / \mathrm{n}),{ }^{58} \mathrm{Ni}(11.56 \mathrm{MeV} / \mathrm{n})\right]$ with different angles of incidence in micas (Biotite, Phlogopite) and soda lime are reported.

\section{Detectors}

In the present work, the natural micacious mineral detectors [Biotite \& Phlogopite] were collected from Nilore mica belt, India, while the soda-lime glass detector was procured from polar industrial corporation, Bombay, India. In these dark micas (Biotite \& Phlogopite), all octahedrons are occupied with cations such as $\mathrm{Mg}$ and Fe. The schematic structure of Phlogopite is shown in Figure $1[4,5,12]$. Glass detector play an important role in track study due to particular advantages such as discrimination of light charged particle, freedom of mechanical rigidness, use prone to environmental change and aging effects [17].

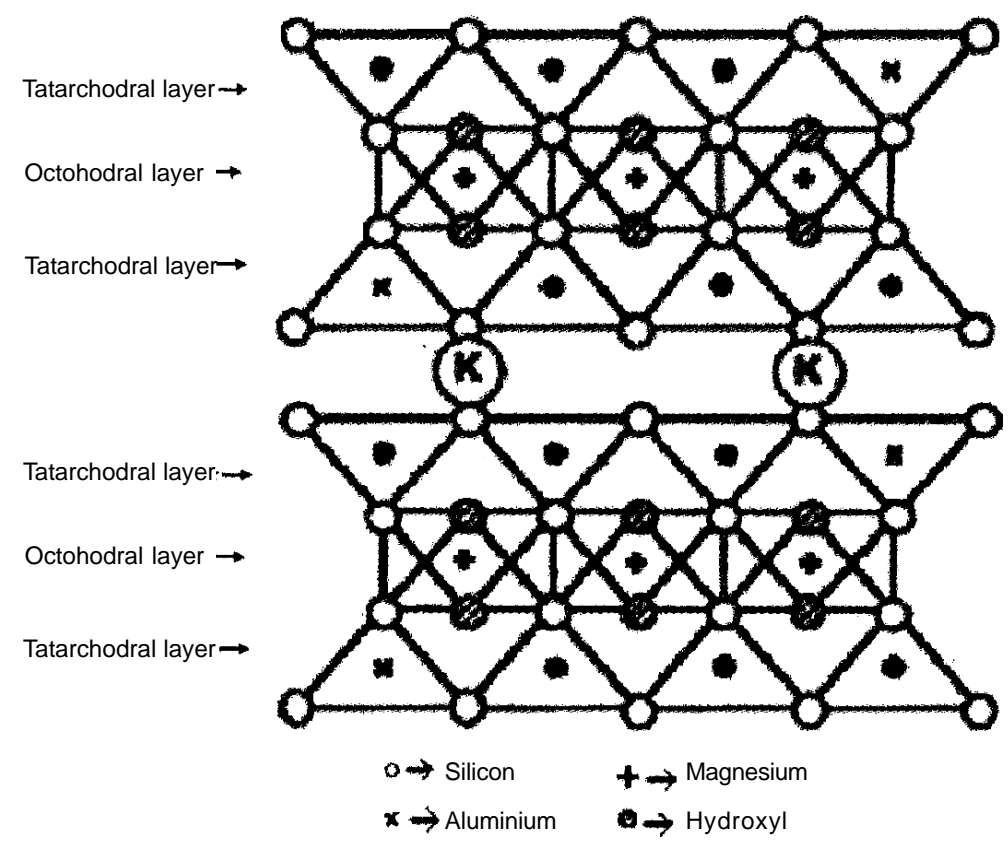

Figure 1. Schematic diagram of phlogopite mica $\left(\mathrm{KMg}_{6} \mathrm{Si}_{6} \mathrm{Al}_{2} \mathrm{O}_{20}(\mathrm{OH}, \mathrm{F})_{4}\right)$. 


\section{Experimental details}

Freshly cleaved thin sheets $(\sim 250 \mu \mathrm{m})$ of biotite and phlogopite micas were cut into a number of disks of $3 \mathrm{~cm}$ diameter each. The basal plane [001] of the samples were irradiated with ${ }^{136} \mathrm{Xe} \quad(11.56$ $\mathrm{MeV} / \mathrm{n}$ ) ions at two different angle of incidence $\left(45^{\circ}\right.$ and $\left.90^{\circ}\right)$ from UNILAC heavy ion accelerator at GSI Darmstadt, Germany. Samples of soda-lime glass detector were also irradiated with heavy ions $\left[{ }^{58} \mathrm{Ni}(11.56 \mathrm{MeV} / \mathrm{n})\right.$, $\left.{ }^{197} \mathrm{Au}(11.64 \mathrm{MeV} / \mathrm{n})\right]$ at the same angles of incidence $\left(45^{\circ}, 90^{\circ}\right)$. The ion fluence in each exposure was kept low $\left(10^{4}\right.$ ions $\left./ \mathrm{cm}^{2}\right)$ to avoid the overlapping of etched tracks. The different irradiated samples were then etched with $40 \% \mathrm{HF}$ acid at constant temperature of $27^{\circ} \mathrm{C}$ for different etching times (4 min, $8 \mathrm{~min}, 12$ min). These etched samples were scanned under the digital optical microscope (BIOLUX, with Scalar 1.0E).

\section{Results and discussion}

The optical images of the heavy ions irradiated micas (Biotite and Phlogopite) and soda-lime glass with different energies and different angles of incidence are shown in the Figures (2-6). It is observed from these images that there are regular hexagonal tracks and irregular polygonal

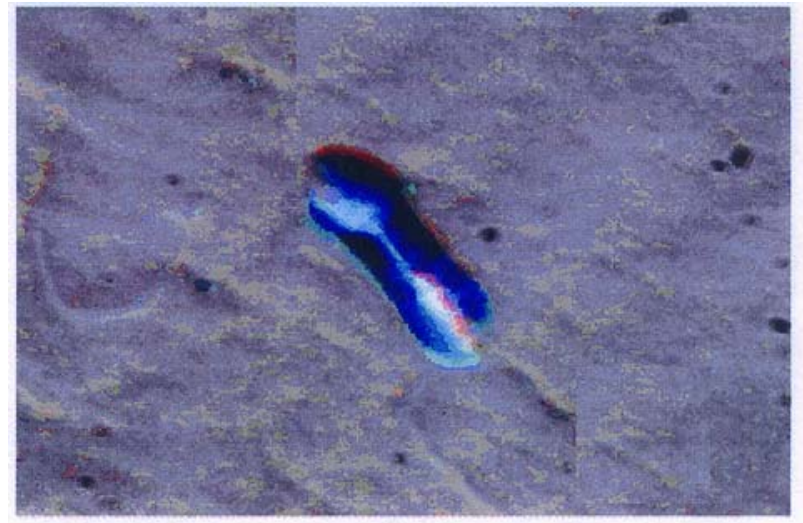

(a)

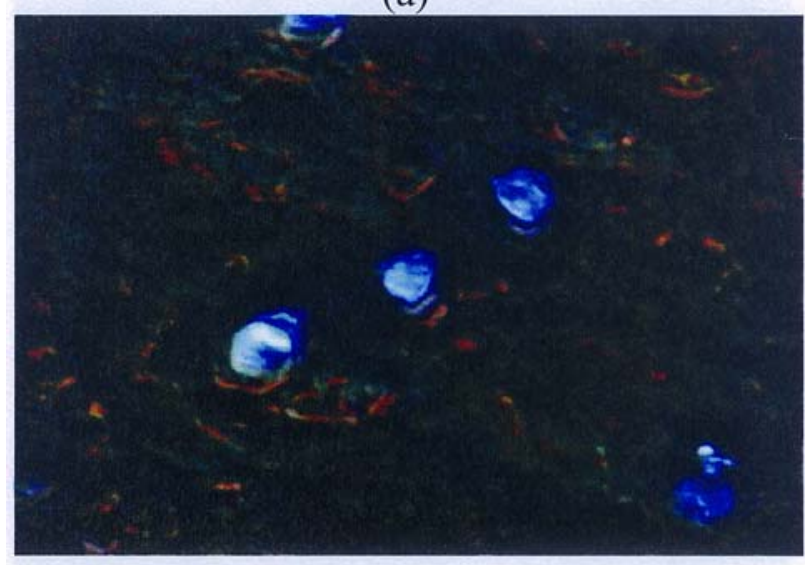

(b)

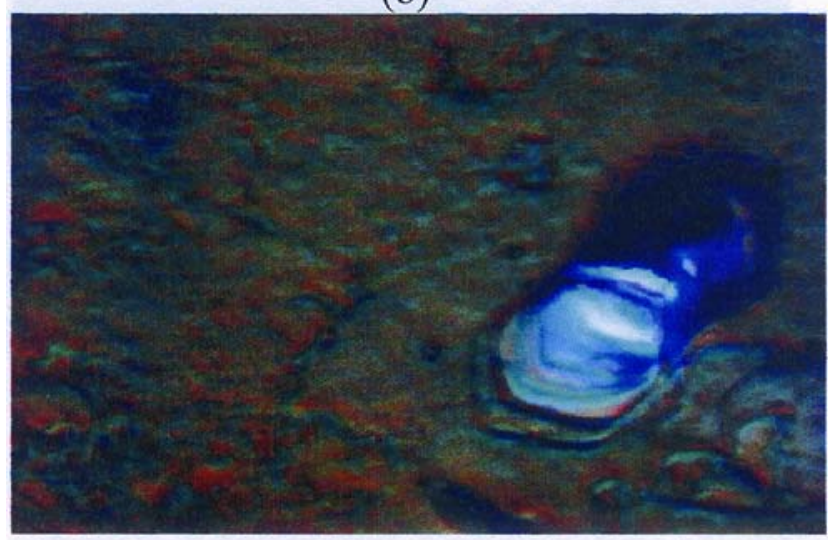

(c)

Figure 2. Optical images of heavy ions $\left[{ }^{136} \mathrm{Xe}(11.56 \mathrm{MeV} / \mathrm{n})\right]$ tracks in biotite mica incident at $45^{\circ}$ and etched in $40 \% \mathrm{HF}$ at room temperature $\left(27^{\circ} \mathrm{C}\right)$ for etching times (a) $4 \mathrm{~min}$, (b) $8 \mathrm{~min}$ and (c) $12 \mathrm{~min}$ at magnification of $400 \mathrm{X}$. 


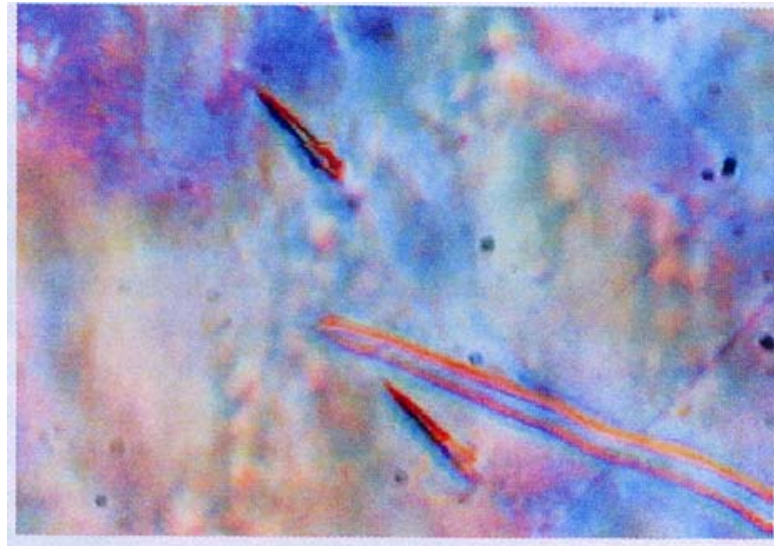

(a)

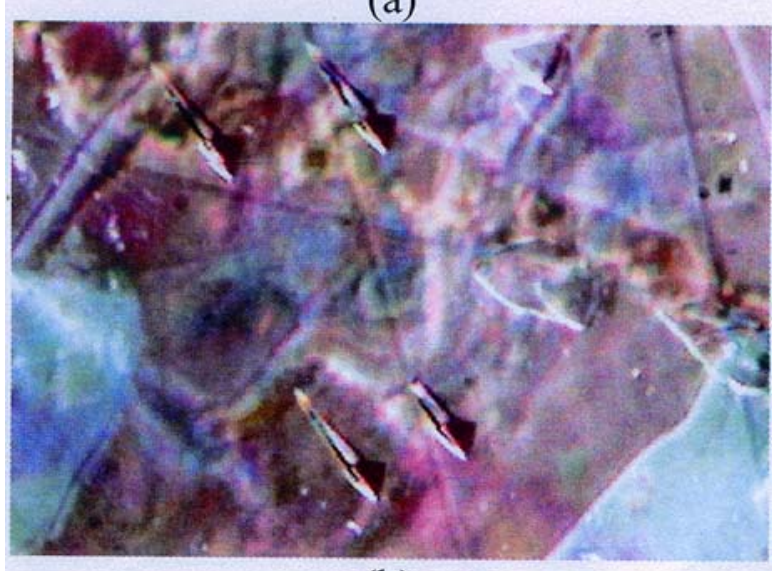

(b)

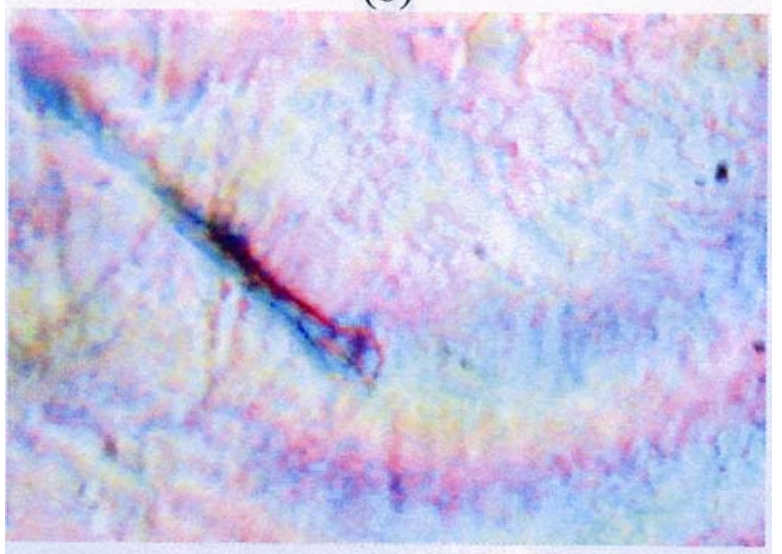

(c)

Figure 3. Optical images of heavy ions [ $\left.{ }^{136} \mathrm{Xe}(11.56 \mathrm{MeV} / \mathrm{n})\right]$ tracks in phlogopite mica incident at $45^{\circ}$ and etched in $40 \% \mathrm{HF}$ at room temperature $\left(27^{\circ} \mathrm{C}\right)$ for etching times (a) $4 \mathrm{~min}$, (b) 8 min and (c) $12 \mathrm{~min}$ at magnification of 400X. Triangular etch pits are also shown in (b). tracks in Biotite. Hexagonal tracks are observed for ions having dip angle $90^{\circ}$ and and for inclined ions $\left(45^{\circ}\right)$ at higher etching time. The irregular polygons geometries shift towards regular hexagonal track geometries with the increase of etching time (Figures 2 and 3). These optical images show that there is a triangular track along with the irregular polygons track geometries in Phlogopite mica (Figure 3b). The observation of optical images in biotite and Phlogopite leads to the conclusion that hexagonal tracks are formed by energetic ions entering the detector at dip angle $90^{\circ}$, polygonal tracks are formed by energetic ions entering the detector at dip angle $45^{\circ}$, and there is a shift of irregular polygonal track opening $\left(45^{\circ}\right.$ angle of incidence) to regular hexagonal shape with the increase of etching time.

The orthogonally incident heavy ions leads to etched track hexagonal contour. Our observations support that, these types of hexagonal structure arise due to the natural hexagonal symmetry of the tetrahedral sheet. The triangular track observed in Figure $3 b$ is a result of low energy fission fragments. These triangular structures are due to the natural triangular symmetry of the 
octahedral sheet. The variation of track geometries along the track is the result of discontinuity in the etchable radiation damage formation along the tracks.

Irregular polygonal track geometries are produce when dip angle is not equal to $90^{\circ}$. The range of the heavy ions and therefore the associated stopping power (or damage) vary strongly with the angle of incidence to the basal plane [12]. Such a dip angle dependence of the stopping power can affect the geometry of the polygonal tracks. If polygonal tracks were heavily etched, most of the polygonal tracks would change to the hexagonal tracks (Figures 2-4). This is analogous to the case of the isotropic detector where elliptical track opening becomes circular when the third phase of the track etching is reached [11].

Figures 5 and 6 show the track opening geometries of various heavy ions $\left[{ }^{58} \mathrm{Ni}(11.56\right.$ $\left.\mathrm{MeV} / \mathrm{n}),{ }^{197} \mathrm{Au}(11.64 \mathrm{MeV} / \mathrm{n})\right]$ in soda-lime glass at different angles of incidence $\left(45^{\circ}\right.$ and $90^{\circ}$ ) with respect to the surface of the glass detector. These optical images are taken at different etching time and at 400X magnification using digital optical microsocope. This shows the circular and elliptical track opening geometries of the heavy

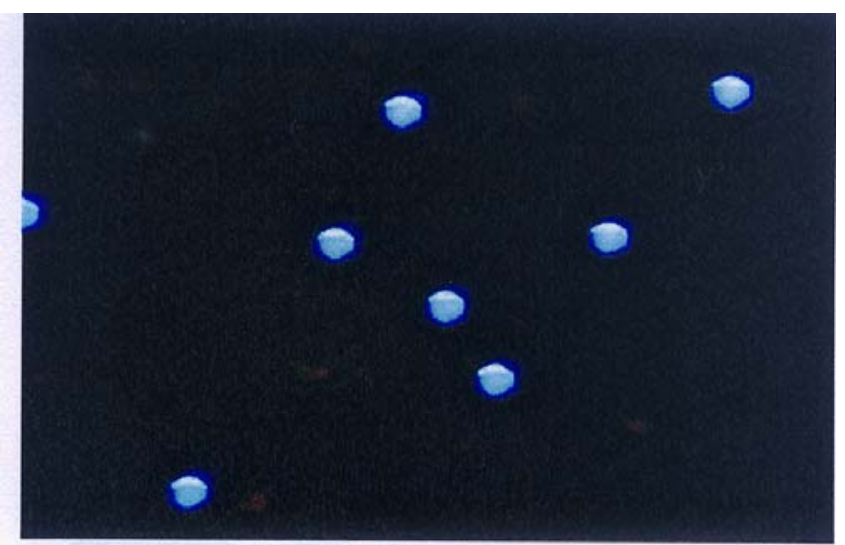

(a)

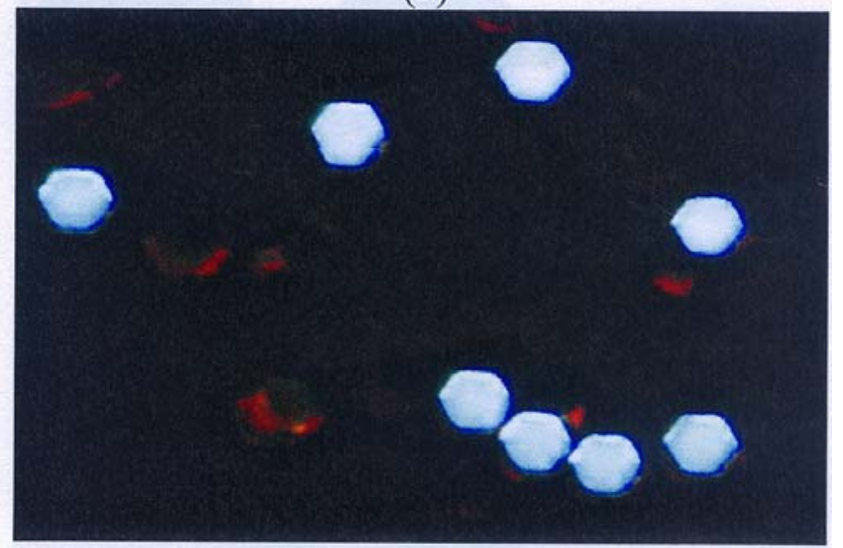

(b)

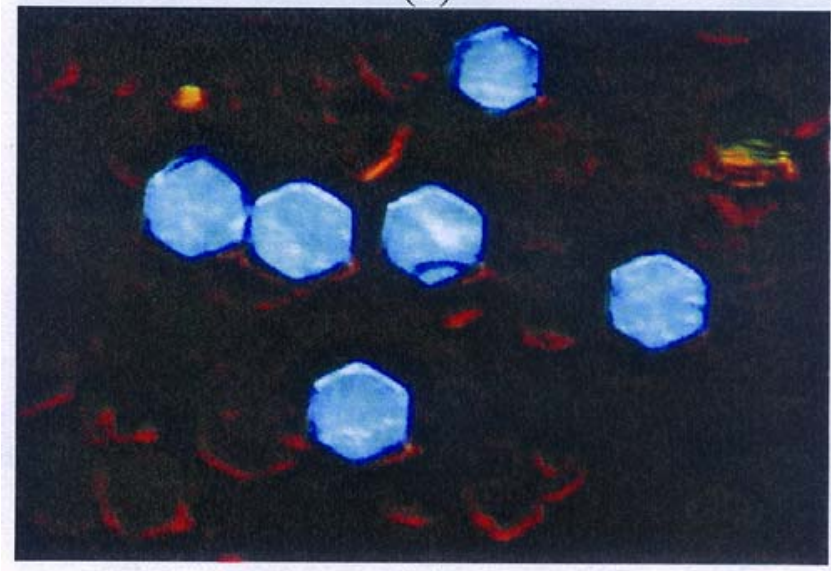

(c)

Figure 4. Optical images of heavy ions $\left[{ }^{136} \mathrm{Xe}(11.56 \mathrm{MeV} / \mathrm{n})\right]$ tracks in biotite mica incident at $90^{\circ}$ and etched in $40 \% \mathrm{HF}$ at room temperature $\left(27^{\circ} \mathrm{C}\right)$ for etching times (a) $4 \mathrm{~min}$, (b) $8 \mathrm{~min}$ and (c) $12 \mathrm{~min}$ at magnification of $400 \mathrm{X}$. 
ion tracks. As Figure shows the track opening geometries change from circular to elliptical as the angle of incidence change from $90^{\circ}$ to $45^{\circ}$. The track in amorphous detectors does not clearly focus on the structure of the detector as compared to the track registration in crystalline detectors.

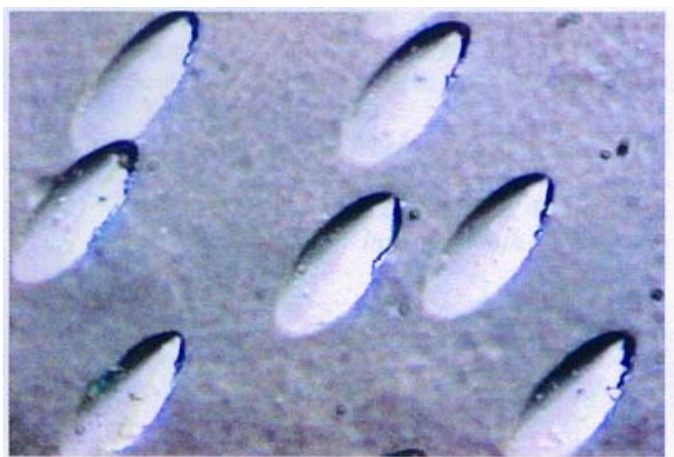

(a)

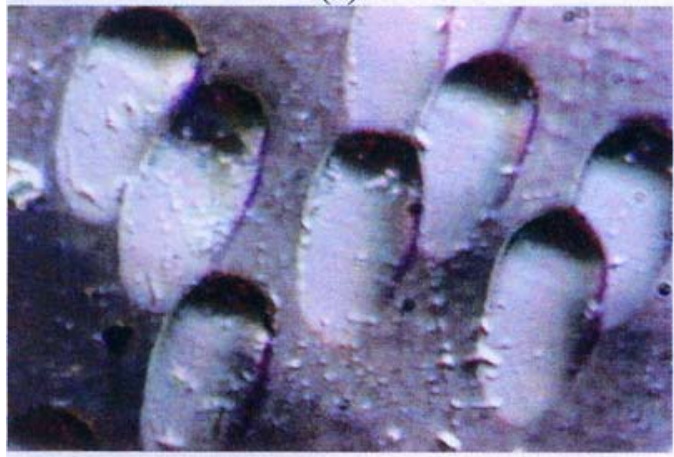

(b)

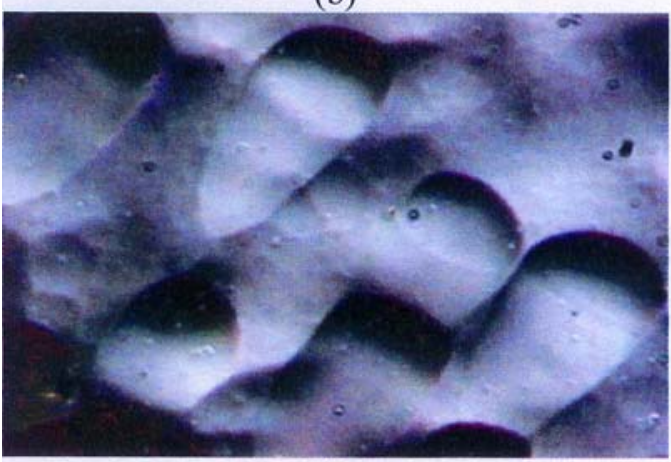

(c)

Figure 5. Optical images of heavy ions $\left[{ }^{58} \mathrm{Ni}(11.56\right.$ $\mathrm{MeV} / \mathrm{n})$ ] tracks in soda-lime glass detector incident at $45^{\circ}$ and etched in $40 \% \mathrm{HF}$ at room temperature $\left(27^{\circ} \mathrm{C}\right)$ for etching times (a) $4 \mathrm{~min}$, (b) $8 \mathrm{~min}$ and (c) $12 \mathrm{~min}$ at magnification of $400 \mathrm{X}$.
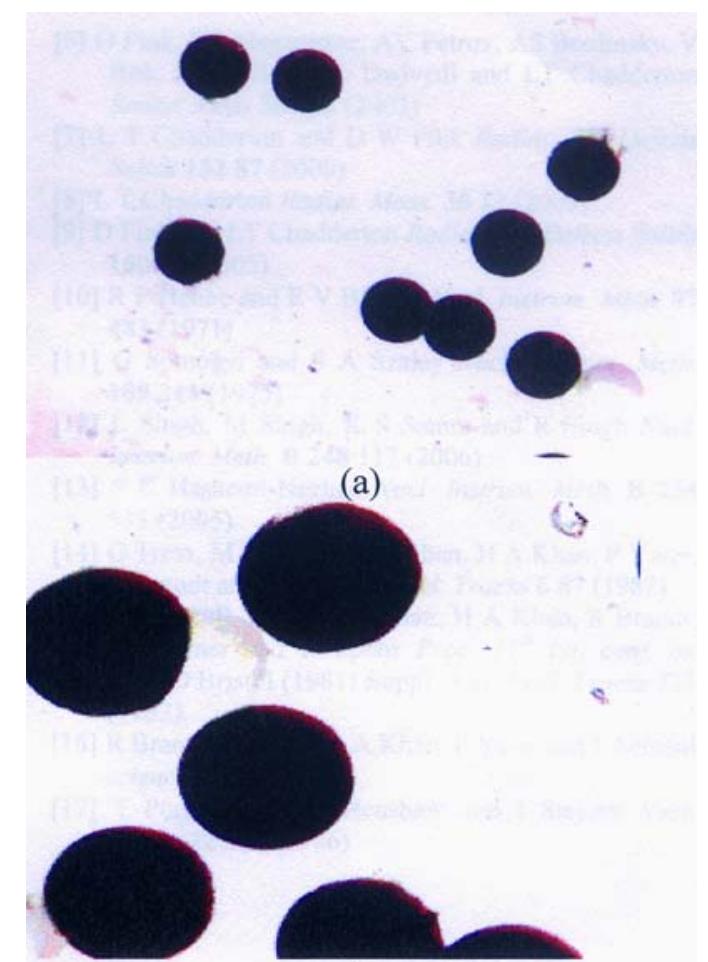

(b)

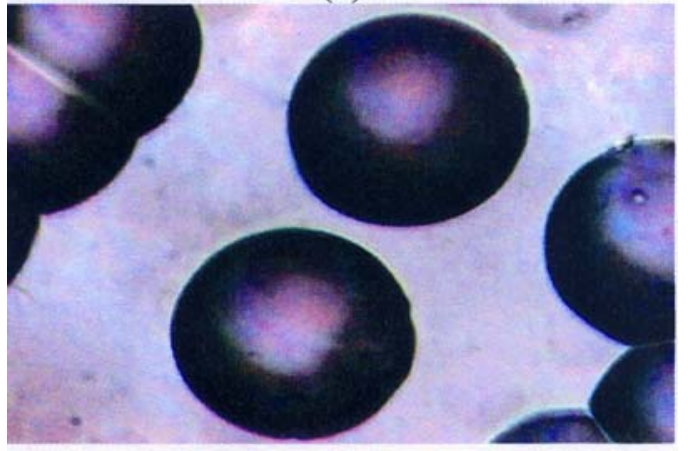

(c)

Figure 6. Optical images of heavy ions $\left[{ }^{197} \mathrm{Au}(11.64\right.$ $\mathrm{MeV} / \mathrm{n})]$ tracks in soda-lime glass incident at $90^{\circ}$ and etched in $40 \% \mathrm{HF}$ at room temperature $\left(27^{\circ} \mathrm{C}\right)$ for etching times (a) $4 \mathrm{~min}$, (b) $8 \mathrm{~min}$ and (c) $12 \mathrm{~min}$ at magnification of $400 \mathrm{X}$. 


\section{Conclusions}

The ion track geometries are found to be related with various target-projectile parameters (viz : type of projectiles, energy, stopping power, angle of incidence of the projectile, density of defects and its reactivity with etchant, etching conditions and chemical structure of the detectors). The density of the radiation damage is related to the stopping power which depends on the angle of incidence of the projectile in anisotropic medium (micas: Biotite and Phlogopite in present case): in turn the shape of the etched track in a particular etchant depends on the angle of incidence of the projectile. Different geometries (Hexagonal, irregular polygon, triangular in case of Biotite and Phlogopite, and circular \& elliptical in case of soda lime glass detector) of heavy ion tracks at different angle of incidence are related to the anisotropic and isotropic behaviour of these detectors.

\section{References}

[1] R L Fleischer, P B Price and R M Walker Nuclear Tracks in Solids (Univ. of California Press, Berkeley, California) (1975)

[2] D Snowden-Ifft, P B Price, L A Nagahara and A Fujishima Phys. Rev. Lett. 702348 (1993)

[3] M Seider, U D Schwarz and R Wiesendanger Phys. Rev. B53 R16 180 (1996)

[4] S R Hashemi-Nezhad Nucl. Instrum. Meth. B142 98 (1998)

[5] M Lang, U A Glasmacher, B Moine, R Neumann and G A Wagner Nucl. Instrum. Meth. B218 466 (2004)

[6] D Fink, P S Alegaonkar, A V Petrov, A S Berdinsky, V Rao, M Müller, K K Dwivedi and L T Chadderton Radiat. Meas. 36605 (2003)

[7] L T Chadderton and D W Fink Radiat. Eff. Defects Solids 15287 (2000)

[8] L T Chadderton Radiat. Meas. 3613 (2003)

[9] D Fink and L T Chadderton Radiat. Eff. Defects Solids 16067 (2005)

[10] R P Henke and E V Benton Nucl. Instrum. Meth. 97483 (1971)

[11] G Somogyi and S A Szalay Nucl. Instrum. Meth. 109211 (1973)

[12] L Singh, M Singh, K S Samra and R Singh Nucl. Instrum. Meth. B248 117 (2006)

[13] S R Hashemi-Nezhad Nucl. Instrum. Meth. B234 533 (2005)

[14] G Trees, M Ellinger, E U Khan, H A Khan, P Vater, R Brandt and M Kadner Nucl. Tracks 687 (1982)

[15] G Trees, P Vater, E U Khan, H A Khan, R Brandt, M Kadner and R Spohr Proc. 11th int. conf. on SSNTD Bristol (1981) Suppl. 3 to Nucl. Tracks 723 (1982)

[16] R Brandt, G Trees, H A Khan, P Vater and J Aerosol Science 14228 (1964)

[17] T Portwood, D L Henshaw and J Stejney Nucl. Tracks 12109 (1986) 